Amir Hossein Mahvi ${ }^{1}$, Hassan Keramati ${ }^{2}$, Bigard Moradi $^{3}$, Rokhsane Hosseini Pouya ${ }^{4}$, Yadollah Fakhri ${ }^{5}$

1. Department of Environmental Health Engineering, Faculty of Health, Tehran University of Medical Sciences, Tehran, Iran

2. Department of Environmental Health Engineering, School of Public Health, Semnan University of Medical Sciences, Semnan, Iran

3. Department of Health Public, Kermanshah University of Medical Sciences, Kermanshah, Iran

4. Food Health Research Center, Hormozgan University of Medical Sciences, Bandar Abbas, Iran

5. Department of Environmental Health Engineering, Student Research Committee, Shahid Beheshti University of Medical Sciences ,Tehran, Iran

*Corresponding Author: Environmental Health Engineering Department, Student Research Committee, Shahid Beheshti University of Medical Sciences ,Tehran, Iran

Tel: 0921-6737245

E-mail:Ya.Fakhri@Gmail.Com

\section{Health Risk Assessment Induced by Inhalation Radon Content in the Indoor Air of Decorative Stone of Storehouses}

Received: 25 Jun. 2016 ; Accepted: 1 Jul. 2017

\section{Abstract}

Background: Radon is a colorless, odorless half-life radioactive gas that that can be emission from decorative stones such as granite, marble, etc. inhaling radon gas over a long period may increase in lung cancer among patients.

Material and Methods: In this cross-sectional-descriptive study, Radon 222 and Thoron concentrations was measured in four Decorative stones of warehouse by Radon meter portable RTM1688-2 model in three stages. In total, 24 concentrations of 24 hours of indoor air and 24 concentrations of 4 hours of Radon 222 and thoron of the background air were measured. Then, effective dose received of Radon 222 and Thoron was calculated by UNSCEAR equations.

Results: The mean indoor air radon and background air were $74 \pm 37$ and $34 \pm 16 \mathrm{~Bq} / \mathrm{m}^{3}$, respectively. The mean concentration of Radon of indoor air in Decorative stones of warehouses in DSW1, DSW2, DSW3 and DSW4 is $72.50 \pm 34,98.25 \pm 43,34.42 \pm 18$ and $88.92 \pm 51 \mathrm{~Bq} / \mathrm{m}^{3}$. The mean effective dose received by the staff from Radon 222 and Thoron at 8 working hours is $0.53 \pm 0.18$ and $0.05 \pm 0.03 \mathrm{mSv} / \mathrm{y}$ and in 16 working hours is $1.05 \pm 0.36$ and $0.11 \pm 0.07 \mathrm{mSv} / \mathrm{y}$, respectively. Also, the mean effective dose received by staff from Radon at 8 and 16 working hours is $0.58 \pm 0.2$ and $1.16 \pm 0.41 \mathrm{mSv} / \mathrm{y}$.

Conclusions: Mean radon concentration in indoor air and the mean effective dose received by staff is lower than the standards level. Decorative stone of warehouses is the resources accumulation of Radon gas that can be reduced by doing corrective actions.

Keywords: Radon 222, Thoron, Effective dose, Decorative stone and warehouses 


\section{ارزيابى خطر سلامت ناشى از استنشاق رادن هواى داخل

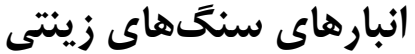

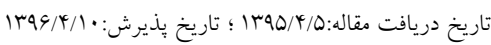

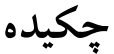

زمينه و هدف: رادن يك كاز بىرنگ، بىبو و راديواكتيو است كه مىتواند از سنگهاى زينتى مانند كرانيت، مرمر و غيره

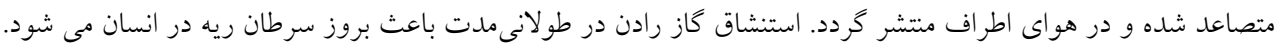

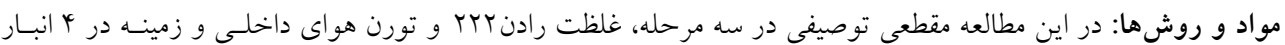

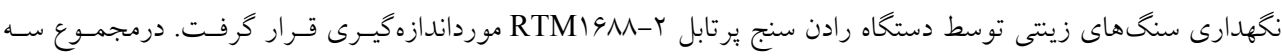

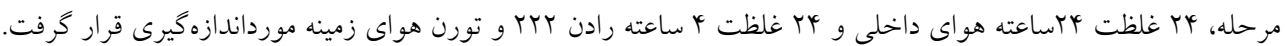
سبس دز مؤثر دريافتى توسط معادلات UNSCEAR محاسبه شد.

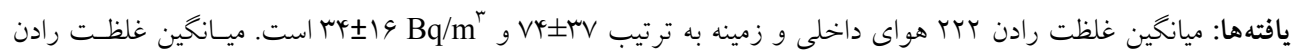

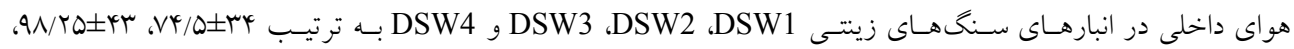

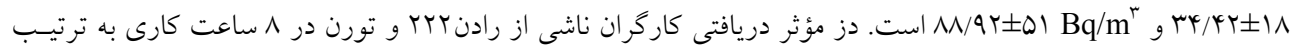

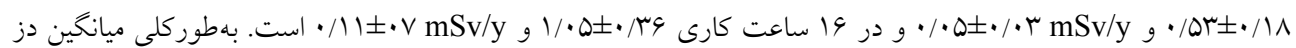

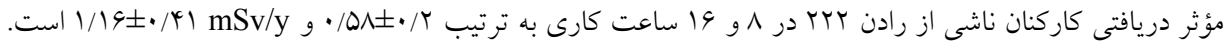

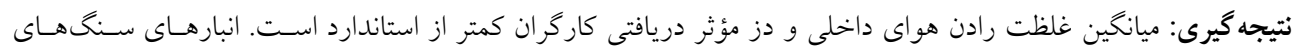

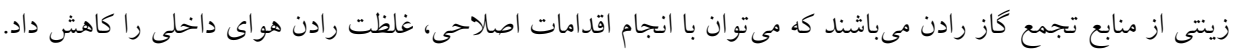
كلمات كليدى: رادن بrا، تورن، دز مؤثر، انبارهاى سنگ هاى زينتى

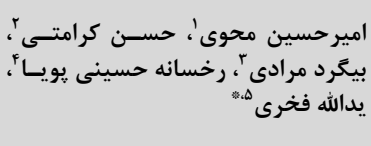

'استاديار كروه مهندسى بهداشت محسيط،

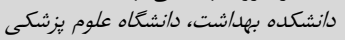
تهران، تهران، ديران

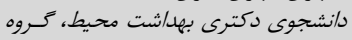

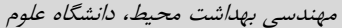
بزرشكى سمنان، سمنان، /يران مجنان

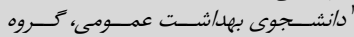

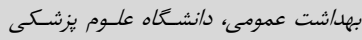
كرمانشاه، كرمانشاه، ايران

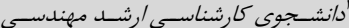

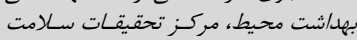

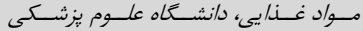
هرمزكان، بندرعباس، /يران

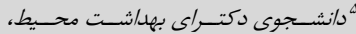

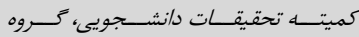

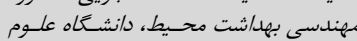
بزشكى شهيد بهشتى، تهران، /يران 


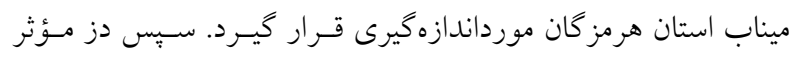

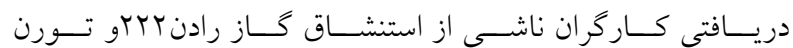

موردمحاسبه و با حدود استاندارد مورد مقايسه كيرد.

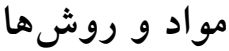

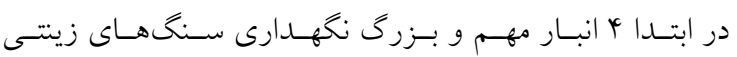

(كرانيت، مرمر و غيره) در شهر ميناب انتخاب كرديد. انــازه خيـرى

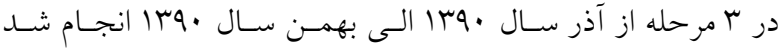

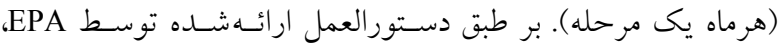
براى اندازه كيرى غلظت رادن هواى داخلى حداقل بايد به مدت بY

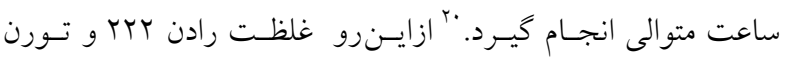

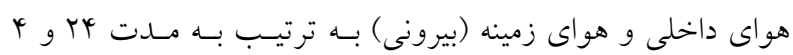

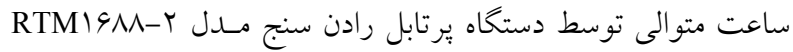
ساخت شركت SARAD كشور آلمان اندازهيـرى شـد. حساسـيت

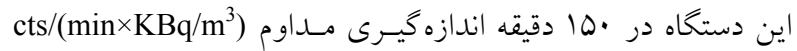

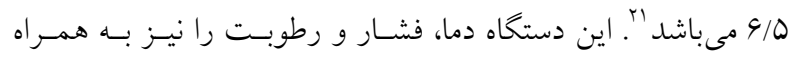

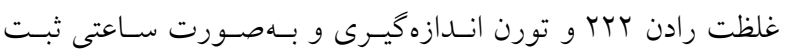
مى كند. حساسيت بالا به همراه آناليز طيفسنجى آلفا، منجر به زمان

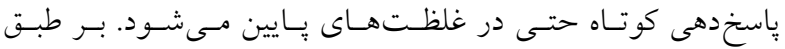
دستور العمل انــازه كيـرى ارائسهشـــه توسط شـركت SARAD، در

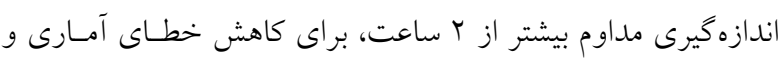

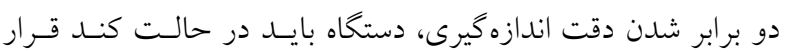

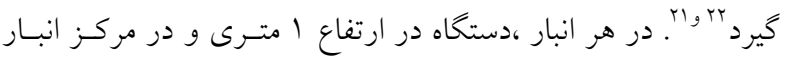

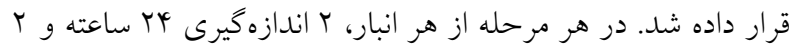

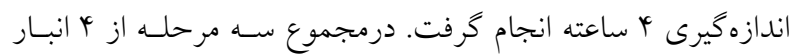

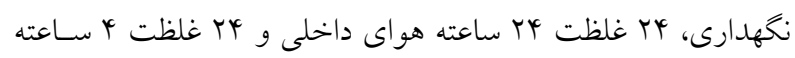

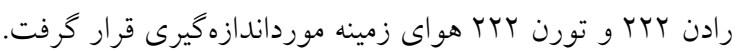
دز مؤثر دريافتى سالانه ناشى از رادن Y ب هواى داخلى، توسط معادله UNSCEAR محاسبه گرديد ( معادله ()؛ $\mathrm{E}_{\mathrm{Rn}}=\mathrm{C}_{\mathrm{n}} \cdot / 4 \times \mathrm{T} \times 9 \times 1 \cdot{ }^{-9}$

در اين معادله E

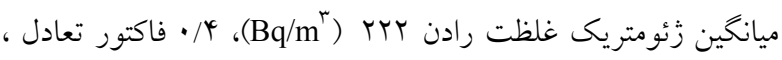

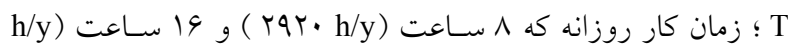

رادن يك كاز بىرنگ، بىبو و راديواكتيو است كه مسىتوانـا از

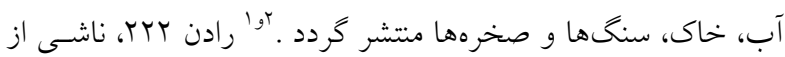
واياشى راديوم צrrدر زنجيره اورانيوم ^بr و تورن ناشى از واياشى

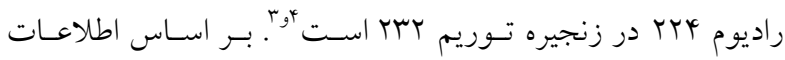
ارائهشده توسط برد بين المللسى حفاظـت در برابـر برتــ ( National Radiation Protection Board

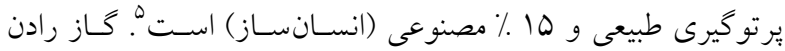

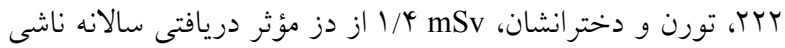
از برتو گيرى طبيعى (T/Y mSv) را به خود اختصاص دادهاند (بيش از •ه./)

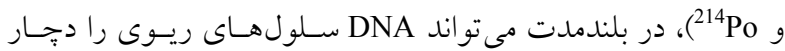

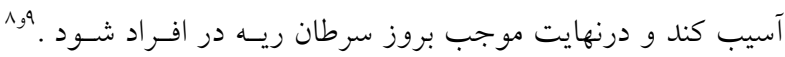
بعد از سيخار دومين علت مرگومير ناشى از سرطان ريه، گَاز رادن

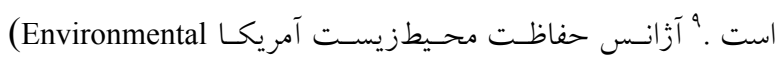
ميزان مرگومير ناشسى از رادن هـواى Protection Agency) (EPA)

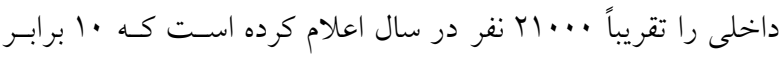

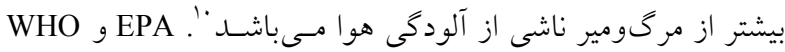

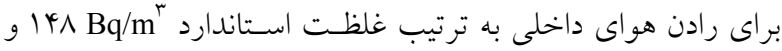

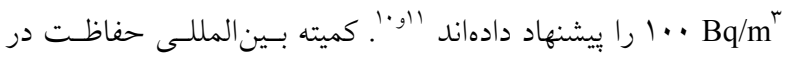
برابر يرتو( ICRP) حداكثر دز مؤثر دريافتى سالانه كاركنان ناشسى از

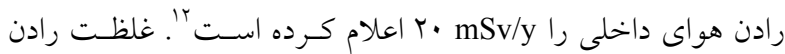

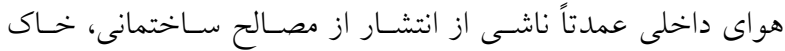

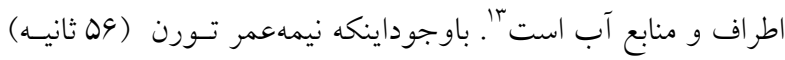

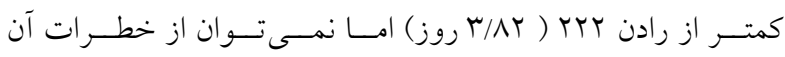
به خصوص در مكانهاى بسته ( انبارها و غيره) جششميوشى كـردو و+1". در سالهاى اخير مطالعات متعددى درزمينه ميـزان انتشـار خـاز

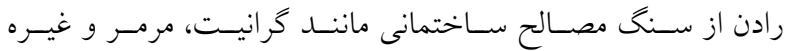

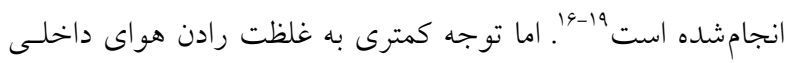

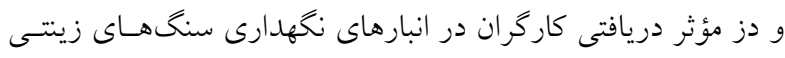

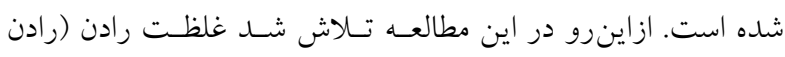

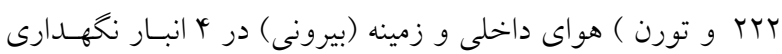
سنخ هاى زينتى (Decorative stones Warehouse (DSW))در شهر 
يافته ها

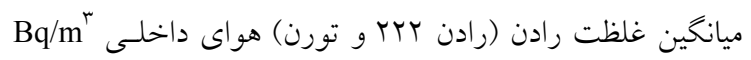

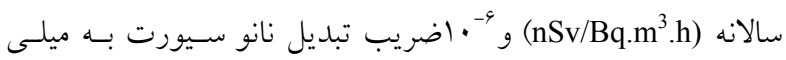

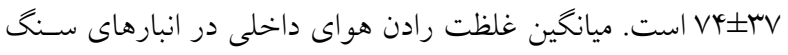

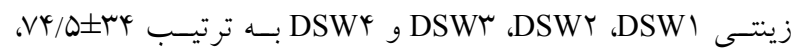

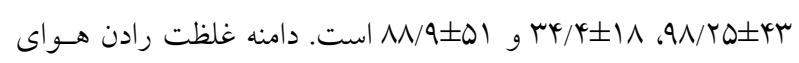

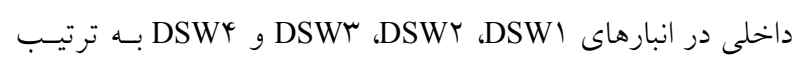

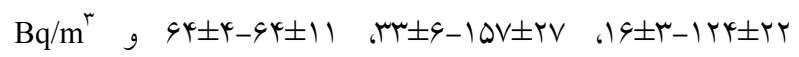
r

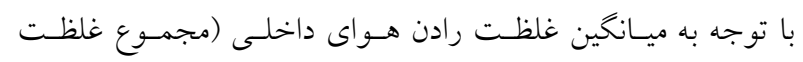
رادن بr و و تـــورن)؛ DSWr<DSW <DSW4<DSWr ميانخين كلسى رادن هـواى بيرونسى

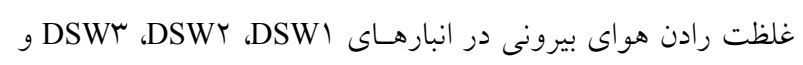

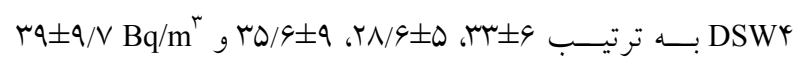

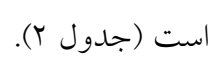

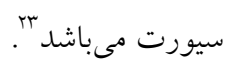
دز مؤثر دريافتى سالانه ناشى از تورن هواى داخلى نيـز توسطط

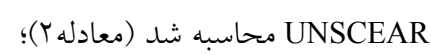
$\left.\mathrm{E}_{\mathrm{Tn}}=\mathrm{C}_{\mathrm{Tn}} \times \cdot / \cdot r \times \mathrm{T} \times Y \cdot \times\right) \cdot \cdot^{-9}$ در اين معادلـه E

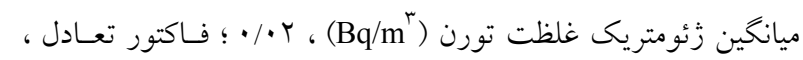

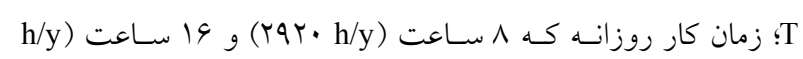

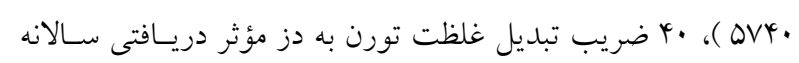
و و (nSv/Bq.m³.h) مىباشد اختلاف غلظت رادن هواى داخلى و بيرونى در ^ انبار نخهدارى

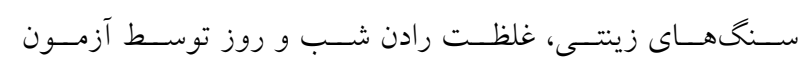
Independent Sample Test

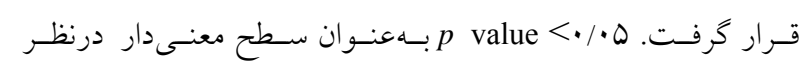
كرفتهشد.

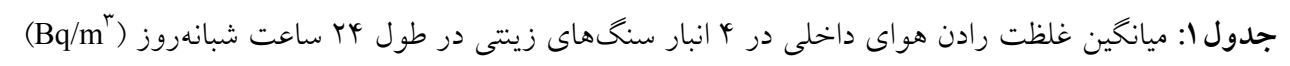

\begin{tabular}{|c|c|c|c|c|}
\hline rDSW & rDSW & rDSW & ${ }^{*} \mathrm{DSW}$ & زمان (ساعت) \\
\hline$\Delta r \pm q$ & $11 \pm 4$ & $\Delta r \pm q$ & ${ }^{* *} r q \pm \emptyset$ & 9 \\
\hline$r \Delta \pm \Lambda$ & $19 \pm r$ & r & $19 \pm r$ & 11 \\
\hline$\mu \pm 0$ & $r \Leftrightarrow \pm \Delta$ & $9 \cdot \pm 11$ & $r \cdot \pm v$ & ir \\
\hline $19 \pm 19$ & $r \wedge \pm 0$ & $r \cdot \pm V$ & $\mu \varphi \pm \Lambda$ & 10 \\
\hline$\Delta \vee \pm 1$ & $19 \pm r$ & $\Lambda V \pm 10$ & $99 \pm 1 r$ & IV \\
\hline$\forall \vee \pm \Lambda$ & $r \cdot \pm r$ & $1.9 \pm 19$ & $V Y \pm \| r$ & 19 \\
\hline$v g \pm 1 r$ & $\forall \bullet \pm \wedge$ & IrrEYr & $\Lambda \perp \pm 10$ & rI \\
\hline IrGDYr & $\forall \notin \pm \Lambda$ & $|r| \pm r r$ & $99 \pm 1 \wedge$ & r \\
\hline$|r| \pm r \mid$ & $G Y \pm 11$ & $1 r q \pm r 4$ & $|\cdot r \pm| \Lambda$ & 1 \\
\hline $\mid V r \pm r$. & $94 \pm 11$ & $1 \% \Delta \pm r \Delta$ & $\| V \pm r \mid$ & $r$ \\
\hline $\mid \wedge r \pm r r$ & $4 q \pm q$ & $1 Q V \pm r V$ & $\mid r Y \pm r r$ & 0 \\
\hline $94 \pm 11$ & $r q \pm 0$ & $1.9 \pm 19$ & $V A \pm 1 r$ & v \\
\hline$\Delta r / \Lambda \pm q$ & $19 / 0 \pm \psi$ & $9 r \pm 11$ & $\mu \psi / \Delta \pm \Lambda$ & روز \\
\hline IKY $\pm Y Y$ & $r q / r \pm q$ & & $1 \cdots / \Delta \pm 1 \wedge$ & شب \\
\hline$N N / q \pm \Delta 1$ & $M y / 4 \pm 1 \Lambda$ & $Q \Lambda / r \Delta \pm \kappa r$ & $V Y / \Delta \pm \mu r$ & ميانگين \\
\hline
\end{tabular}




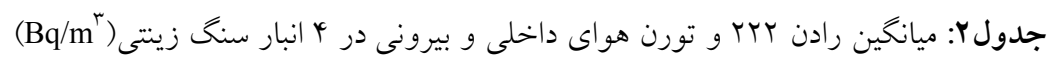

\begin{tabular}{|c|c|c|c|c|c|c|c|c|c|c|c|c|c|c|c|c|}
\hline \multicolumn{4}{|c|}{ مجموع } & \multicolumn{4}{|c|}{ مرحله r } & \multicolumn{4}{|c|}{ مرحله r } & \multicolumn{4}{|c|}{ مرحله 1} & \\
\hline \multicolumn{2}{|c|}{ زمينه } & \multicolumn{2}{|c|}{ داخلى } & \multicolumn{2}{|c|}{ زمينه } & \multicolumn{2}{|c|}{ داخلى } & \multicolumn{2}{|c|}{ زمينه } & \multicolumn{2}{|c|}{ داخلى } & \multicolumn{2}{|c|}{ زمينه } & \multicolumn{2}{|c|}{ داخلى } & \\
\hline تورن & رادن & تورن & رادن & تورن & رادن & تورن & رادن & تورن & رادن & تورن & رادن & تورن & رادن & تورن & رادن & \\
\hline & YYY & & YYY & & YYY & & YYY & & YYY & & YYY & & TrY & & YYY & \\
\hline v & $T \Delta / 9$ & $r \mid / r$ & $01 / 1$ & 19 & 41 & $r \mu$ & Qr & $r$ & TO & $\pi$ & $\Delta \Delta$ & $r$ & 11 & 11 & 40 & DSW \\
\hline$\lambda / r$ & $r \cdot / r$ & $K Y / 1$ & $\Delta \varphi$ & $\wedge$ & 19 & 40 & $\Delta V$ & 11 & 19 & rr & $\Delta V$ & 4 & re & r. & $\Delta t$ & DSWr \\
\hline$V / 9$ & rA & $V / q$ & $r \& / r$ & ir & rq & $\wedge$ & rq & 9 & YY & $\wedge$ & rq & $\varphi$ & $\mu_{1}$ & 9 & ro & DSWr \\
\hline $9 / 9$ & rq & $r \mid / r$ & $q V$ & 11 & $\Delta 9$ & 10 & 99 & 0 & r & ry & 90 & 9 & 19 & 10 & $q V$ & DSWr \\
\hline$\Lambda / r \pm{ }^{\varphi}$ & $r \varphi \pm 1 \wedge$ & $r r / r \pm \mid \psi$ & $0 . \pm I V$ & $\mid r \pm \psi$ & $r q \pm 1 q$ & $r+19$ & $\Delta 1 \pm 1 \wedge$ & $v \pm r$ & $r \cdot \pm \varphi$ & $r V \pm 10$ & $01 \pm 19$ & $\Delta \pm r$ & $r Y \pm q$ & $r \cdot \pm 1 k$ & $\uparrow \wedge \pm \uparrow \wedge$ & ميانخين' \\
\hline
\end{tabular}

دز مؤثر دريافتى كاركنان ناشى از رادن هواى داخلـى انبارهـاى

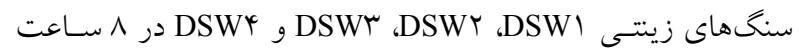
كارى به ترتيب 09/ •، 199•، م/ • و

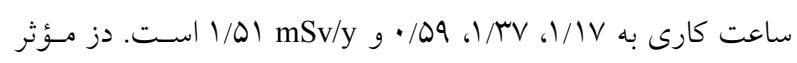

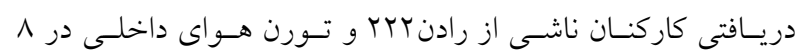

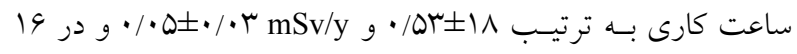

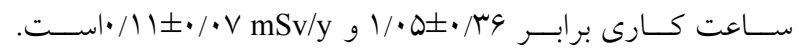

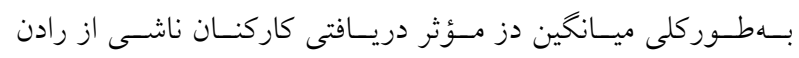

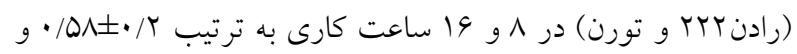
أl mSv/y زيتتى ازلحاظ دز مـؤثر دريـافتى رادن DSWI<DSWr<DSWr

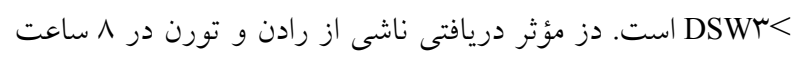
كارى به ترتيب 1// •

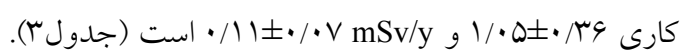

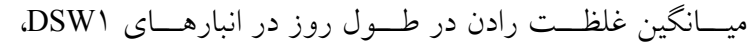
DSWr ( a شـب بـهروز در هــواى داخلـى بــراى انبارهـاى DSW D. DSW،

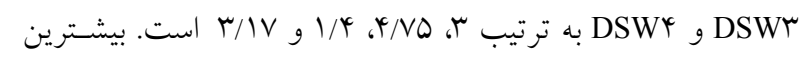
و كمترين نسبت به ترتيب مربـوط بـه انبارهـاى DSWY و

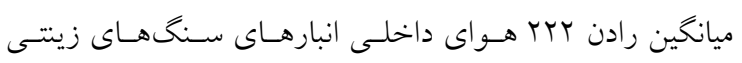
DSW 9V/·V و تسورن بـه ترتيـب

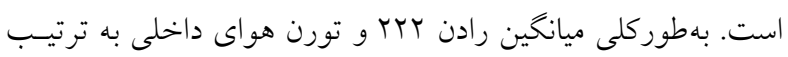

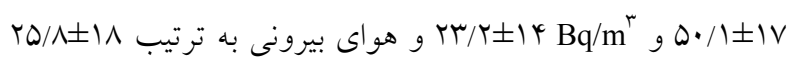
و

\begin{tabular}{|c|c|c|c|c|c|}
\hline \multicolumn{2}{|c|}{ دز مؤثر دريافتى (mSv/y) } & \multirow[b]{2}{*}{ مجموع } & \multirow[b]{2}{*}{ تورن } & \multirow[b]{2}{*}{ رادن Yr Tr } & \\
\hline 19 & $\wedge$ & & & & \\
\hline $1 / 1 V$ & $\cdot / 09$ & $V \psi \pm r \mu$ & $r \mid / r$ & $01 / 1$ & DSWI \\
\hline $1 / T V$ & .199 & $৭ \wedge \pm \vee r$ & $K Y / 1$ & $\Delta S$ & DSWr \\
\hline$\cdot / 09$ & $\cdot / \mu$ & $r_{\wedge \pm \ \wedge}$ & $V / 9$ & $r \& / r$ & DSWr \\
\hline $1 / 01$ & $\cdot / V Q$ & $\wedge \pm \Delta \backslash$ & $r M / r$ & $9 V$ & DSWr \\
\hline$|/| q \pm \cdot / 4 \mid$ & $\cdot / \Delta \wedge \pm \cdot / r$ & $V \mu \pm r v$ & $r \mu / r \pm 1 \%$ & $0 \cdot / 1 \pm 1 \mathrm{~V}$ & ميانخين \\
\hline
\end{tabular}




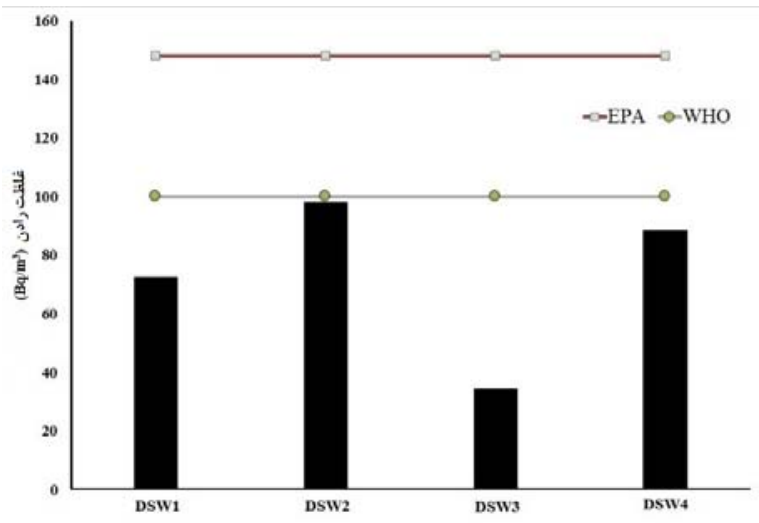

شكل ا: مقايسه ميانحين غلظت رادن هواى داخلى † انبارهاى سنگهاى زينتى (رادن Yr و تورن) با استانداردهاى EPA و WHO

نخهارى سنخ هاى زينتى، اختلاف معنسىدار بـين ميـانخين غلظـت

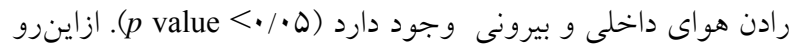

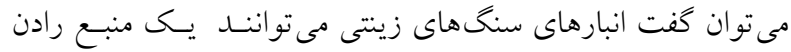
باشند. اين تفاوت غلظت رادن هواى داخلى در انبارهاى سنگ هــاى زينتى ناشى از تفاوت در ميـزان تهويـه هــا (طبيعى و مصـنوعى)،

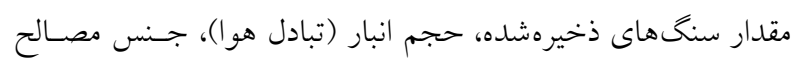

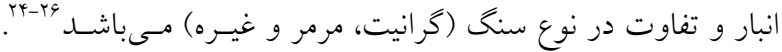

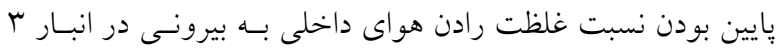

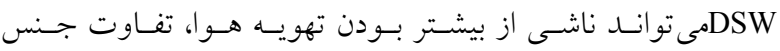
سنخ هاى ذخيرهشده و مصالح بدنه ساختمان انبار باشد. از آنجايى كه تهويه طبيعى و مصنوعى در هنگام شب كمتر از روز است ازايسنرو غلظت رادن هواى داخلى نسبت بهروز نيز بيشـتر اسـت. (ه•/ نشان داد كه غلظت رادن هواى داخلى در شـب بـهصـورت (value

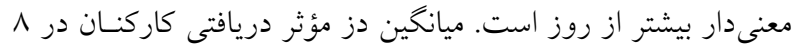

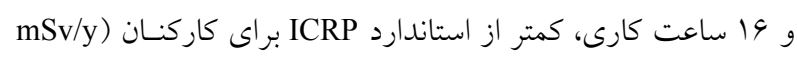

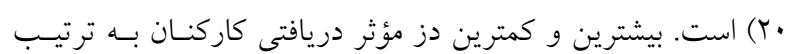

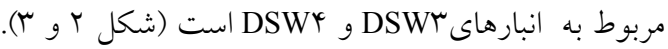
ازآنجايى كه با افزايش زمان مواجهه دز مؤثر نيز افزايش مى يابسـ

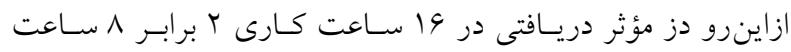

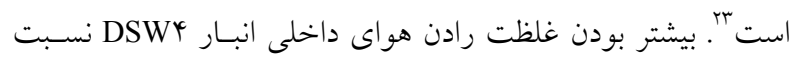

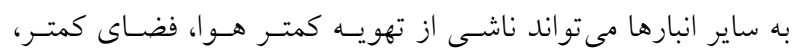
تفاوت در جنس سنگ بهاى به ذخيرهشـده و يـا تفـاوت در مصـالح

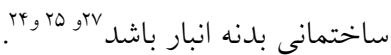

نPA نسبت ميانخين غلظت رادن هـواى داخلسى بـه اسـتاندارد

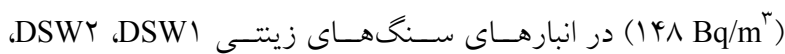

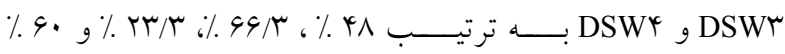
است". ميانخين غلظت رادن هواى داخلى در همه انبارهـا، كمتـر از

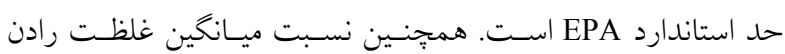

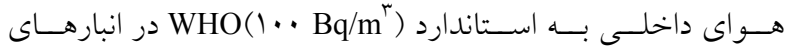

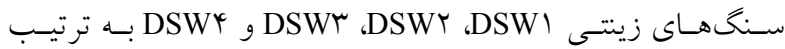

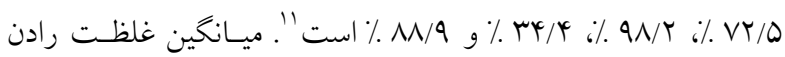
هواى داخلى نيز كمتر از حد استاندارد WHO است (شكل (). نسبت ميانخين غلظت رادن هواى داخلى بـه هـواى بيرونسى در

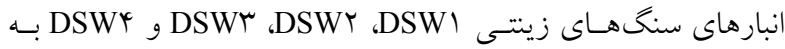

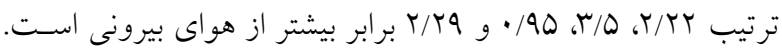
بيشترين و كمترين نسبت غلظت رادن هواى داخلى به هواى بيرونى

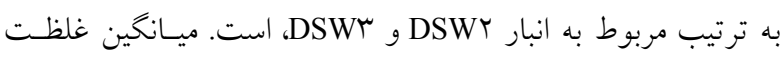

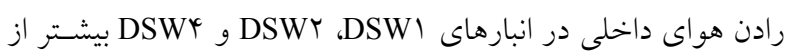
هواى بيرونى و در انبار DSW3 كمتر از هواى بيرونى اسـت. آنـاليز

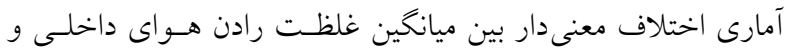

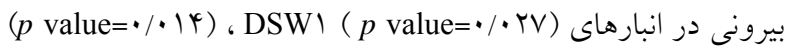

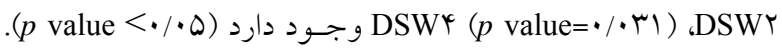
اما اختلاف معنى دار بين غلظت رادن هواى داخلى و بيرونى در انبار

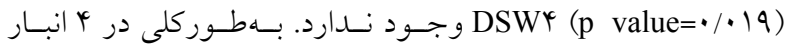




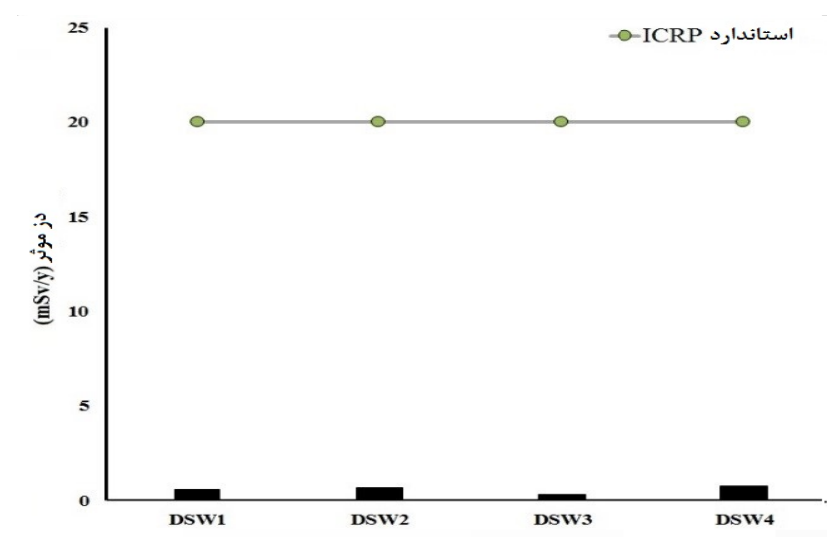

شكل rا: مقايسه دز مؤثر دريافتى كاركنان با استاندارد ICRP ساعت كارى) (1 )

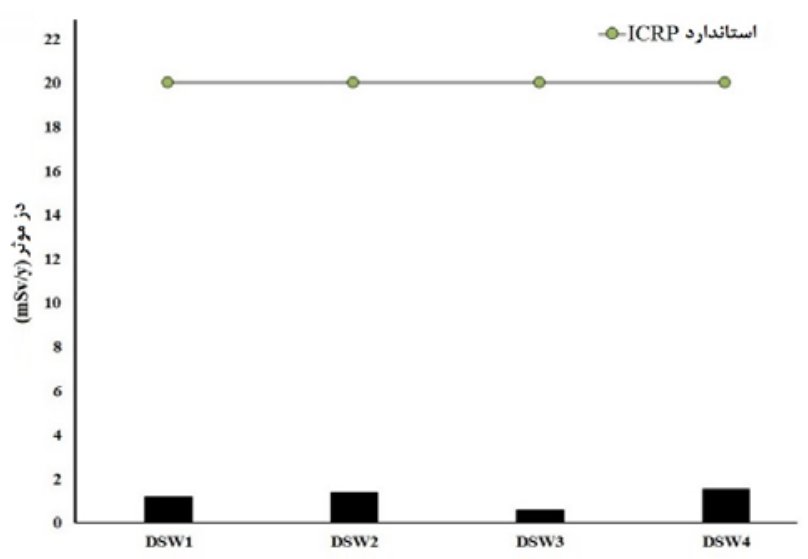

شكل r: مقايسه دز مؤثر دريافتى كاركنان با استاندارد ICRP (19 ساعت كارى)

كاركنان در ^و و 19 ساعت كارى نيز كمتر از حسـ اسـتاندارد ICRP

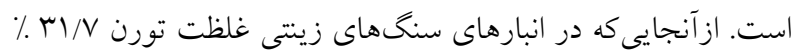

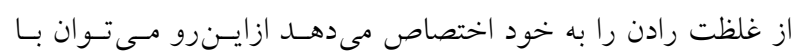

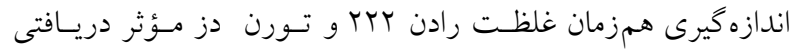

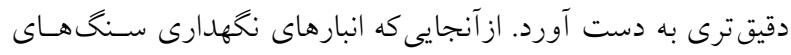

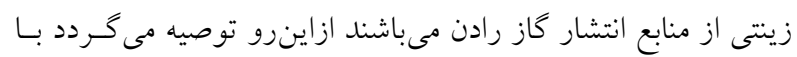
انجام تهويه مناسب، كاهش زمان كارى و ساير روش هاى اصلاحى،

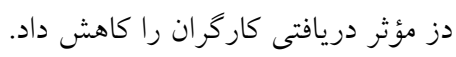

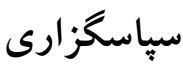

كميته تحقيقات دانشجويى دانشخاه علوم يزشكى شهيد بهشتى

تأمين كننده مالى اين تحقيق مىباشد (74310).
ازاينرو مىتواند در مطالعات بعدى تأثير هر يكى از اين متغيرها

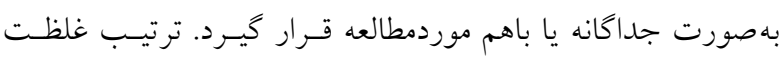

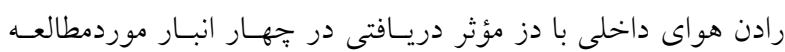
متفاوت است زيــا غلظـت تـورن در انبـار DSWr بيشـتر از انبـار است ازاينرو دز مؤثر دريافتى ناشسى از رادن (رادن TSWF

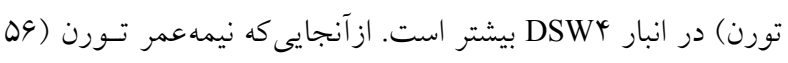

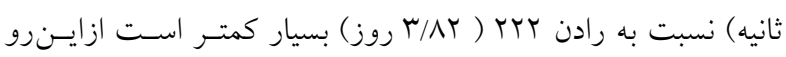
سهم تورن نيز در دز مؤثر دريافتى رادن كمتر است بهاو

\section{نتيجه گيرى}

ميانخين غلظت رادن هواى داخلى انبارهـاى ســخـهـاى زينتى هـ كمتر از حسـود اسـتاندارد WHO و EPA اسـت. دز مـؤثر دريـافتى 


\section{References}

1. Ju y-j, ryu y-h, jang h-c, dong k-r, chung w-k, cho j-h, et al. A study on concentration measurements of radon-222 (uranium series) and radon-220 (thoron series) emitted to the atmosphere from tex (cementitious), red brick, and ecocarat among construction materials. Journal of the korean physical society. 2012;60(7):1177-86.

2. Topçu n, biçak d, çam s, ereeş f. Radon exhalation rate from building materials using cr-39 nuclear track detector. Indoor and built environment. 2013;22(2):384-7.

3. Cohen bs ,xiong jq, fang c-p, li w. Deposition of charged particles on lung airways. Health physics. 1998;74(5):554-60.

4. Cothern cr, smith je. Environmental radon: springer; 1987.

5. Green b, lomas p, luykx f. Natural radiation atlas of europe. Radiation protection dosimetry. 1991;36(2-4):858.

6. Richard cc, e sj. Environmental radon: springer; 1987.

7. Magill j, galy j. Radioactivity radionuclides radiation. Berlin heidelberg newyork: springer; 2005. Available from: www.nuclides.net.

8. Kávási n, somlai j, szeiler g, szabó b, schafer i, kovács t. Estimation of effective doses to cavers based on radon measurements carried out in seven caves of the bakony mountains in hungary. Radiation measurements. 2010;45(9):1068-71.

9. Zeeb h, shannoun f, editors. Who handbook on indoor radon, a public health perspective. Geneva ,switzerland: world health organization; 2009.

10. Environmental protection agency. Consumer's guide to radon reduction. 2010.

11. World health organization. Sets radon action level of 2.7 less lung cancer risk than epa 4.0. Global press release distribution. . 2009.

12. Protection icor, icrp. Icrp publication 66: human respiratory tract model for radiological protection: elsevier health sciences; 1994.

13. Ramasamy $v$, dheenathayalu $m$, ravisankar $r$, ponnusamy $\mathrm{v}$, rajamanickkam gv, sahayam d, et al. Natural radioactivity measurements in beach-rock samples of south-east coast of tamilnadu, india. Radiation protection dosimetry. 2004;111(2):229-35.

14. Anjos rmd, ayub jj, cid as, cardoso r, lacerda t External gamma-ray dose rate and radon concentration in indoor environments covered with brazilian granites. Journal of environmental radioactivity. 2011;102(11):1055-61.

15. Janik m, omori y, yonehara h. Influence of humidity on radon and thoron exhalation rates from building materials. Applied radiation and isotopes. 2015;95:102-7.

16. Righi s, bruzzi 1. Natural radioactivity and radon exhalation in building materials used in italian dwellings. Journal of environmental radioactivity. 2006;88(2):15870 .

17. Nassiri p, ebrahimi h, jafari shalkouhi p. Evaluation of radon exhalation rate from granite stone. Journal of scientific \& industrial research. 2011;70(3):230-1.

18. Kumar a, chauhan $\mathrm{r}$, joshi $\mathrm{m}$, sahoo b. Modeling of indoor radon concentration from radon exhalation rates of building materials and validation through measurements. Journal of environmental radioactivity. 2014;127:50-5.

19. Hosoda m, sorimachi a, yasuoka y, ishikawa t, sahoo sk, furukawa $\mathrm{m}$, et al. Simultaneous measurements of radon and thoron exhalation rates and comparison with values calculated by unscear equation. Journal of radiation research. 2009;50(4):333-43.

20. Environmental protection agency. Epa indoor radon and radon decay product measurement device protocols http://www.epa.gov/radon/pubs/devprot3.htm

21. Ursulean i, corețchi 1, chiruță i, virlan s, editors. Estimation of indoor radon concentrations in the air of residential houses and mines in the republic of moldova. Paper presented at the first east european radon symposium-feras; 2012.

22. Gmbh s. Application note an-003_en: measurement of the radon concentration of water samples june 2007. Available from: www.sarad.de.

23. United nations scientific committee on the effects of atomic radiation (unscear). Sources and effects of ionizing radiation; united nations scientific committee on the effects of atomic radiation. Usa: 2000 .

24. Perrier $f$, richon $p$, crouzeix $c$, morat $p$, le mouël $j-1$. Radon-222 signatures of natural ventilation regimes in an underground quarry. Journal of environmental radioactivity. 2004;71(1):17-32.

25. Alsaedi ak, almayahi b, alasadi a. Cement 222rn and 226ra concentration measurements in selected samples from different companies. Asian journal of natural \& applied sciences (ajsc). 2013;2(4):95-100.

26. Nain $\mathrm{m}$, chauhan $\mathrm{r}$, chakarvarti s. Alpha radioactivity in indian cement samples. Iran j radiat res. 2006;3:1-71.

27. Biira s, kisolo aw, d'ujanga fm. Concentration levels of radon in mines, industries and dwellings in selected areas of tororo and busia districts, eastern uganda. 